\title{
Conduction Mechanism under DC and AC Fields of Methyl Red Dye as a New Organic Semiconductor
}

\begin{abstract}
A. SALEM
Solid State Lab., Physics Department, Faculty of Science, South Valley University, Qena, Egypt

This manuscript studies the AC and DC electrical conductivity of the organic compound methyl red dye, which is intensively examined due to its specific properties. The test samples were characterized by X-ray diffraction analysis. During the performance by the experts, the electrical conductivity of the direct current methyl red dye exhibited almost the symmetrical behavior to that of the general properties of organic semiconductors. The mechanism of conductivity of the alternating current methyl red dye was controlled by a correlative, characteristic barrier hopping model, as confirmed by an observed reduction in activation energy.
\end{abstract}

DOI: 10.12693/APhysPolA.136.952

PACS/topics: organic semiconductor, methyl red, impedance spectroscopy, electrical modulus

\section{Introduction}

Organic semiconductors are the organic materials, whose electrical conductivity falls between insulators and metals. Organic semiconductors have attracted increasing research efforts over the previous decades. In the recent past, major improvements in organic thin-film devices have been explored. Many successful techniques, such as organic light-emitting diodes (OLEDs), organic thin-film transistors, organic photovoltaics (OPVs), organic field-effect transistors (OFETs), and solar cells have provided progress in device performance with expected increase range of applications and daily life usage in the future [1]. These organic semiconductors have many unique electrical, optical, and magnetic properties, which are valuable for nanotechnology, electronics, optics, and other fields of science and engineering technology of materials [2]. Organic semiconductors are a class of materials that are carbon-rich compounds with a structure tailored to optimize a function, such as charge carrier mobility or luminescent properties. Organic electronic materials can be traditionally classified into three main categories: small-molecules, polymers, and biological compounds. Small-molecule is a commonly used term to refer to compounds that have a well-defined molecular weight.

The weight of each molecule of a small-molecule is the same. Alternatively, polymers are long-chain molecules comprised of a varying number of repeat units. The weight of each polymer molecule of the same polymer is different. On the extreme end of the complexity scale are biological molecules, which have yet to find a clear application in optical or electronic systems [3]. Computer simulations and modeling contributed substantially to the understanding of the basic physical processes in organic semiconductors [4-7]. Dyes are generally utilized in different industries, such as textiles, leather, plastic, paper, and cosmetics, for coloring their final products [8].

corresponding author; e-mail: aasalem@kku.edu.sa 4-dimethylaminobenzene-2-carboxylic acid (methyl red, $\mathrm{MR}$ ) is an anionic azo dye, commonly used in titrations as $\mathrm{pH}$ indicator, and it is a dark red crystalline powder that turns red in acidic solutions. MR has a conjugated structure, and is rich in $\pi$-electrons $[9,10]$. It has been used in vast quantities for textile and paper dyeing and it causes irritation of the eye, skin, and digestive tract if inhaled/swallowed [11-12].

This paper focuses on these materials as a new semiconductor for their possible electronic applications towards technology specifically rechargeable storage batteries, electrochromic shows and smart windows, light emitting diodes (LEDs), harmful waste cleanup, sensors, erosion inhibitors, field effect transistors (FETs), electromagnetic interference (EMI) shielding, etc. The objective of the present research is to study the structural, $\mathrm{DC}$ and $\mathrm{AC}$ electrical conductivity measurements of MR dye. Structural characterization of MR was performed using X-ray diffraction (XRD) analysis. The MR was in the form of a powder and was converted to cylindrical pellets for electrical measurements.

\section{Experimental procedures}

Methyl red dye (Fig. 1) used is of the highest commercially available purity, purchased from Aldrich Chemical Co. and was used as received. The various samples were compacted by using hydraulic equipment under a pressure of $3 \mathrm{kbar}$ in the form of cylindrical pellets and subjected to impedance spectroscopy analyses. The equipment was a SOLARTRON impedance meter. The tube shaped pellets were set between platinum electrodes, in a particular heating cell, and the cell was introduced in a furnace, under air. All estimations happened in the temperature range of 285-435 K.

$\mathrm{AC}$ electrical measurements were performed using an Agilent E4980A LCR meter at temperatures from 285 to $435 \mathrm{~K}$. The frequency varying between 1 and $10^{7} \mathrm{~Hz}$. Results of impedance analysis show that as prepared methyl red pellet behaves as an n-type semiconductor material. 
<smiles>CN(C)c1ccc(/N=N/c2ccccc2C(=O)O)cc1</smiles>

Fig. 1. Chemical structure of MR organic compound.

The disorder diminished with increment in temperature of the pellet. Analysis of AC conductivity shows that the conduction mechanism in the MR pellet is thermally activated, which can be explained by the correlated barrier hopping $(\mathrm{CBH})$ model. The electrical properties of $\mathrm{MR}$ were studied over a range of frequency and temperature using the complex impedance spectroscopy (CIS). This analysis technique allows to separate the real $Z^{\prime}$ and imaginary $Z^{\prime \prime}$ parts of the impedance $Z$ and hence provides a true picture of the material properties. Semiconductor characterization system from Keithley has been used to measure the DC electrical conductivity of the sample because of their ability to measure small currents. In this setup the sample was considered as the electrical resistance. Across the sample a voltage was applied and corresponding current was noted. Using Ohm's law, the resistance was calculated. Hence, conductivity (the reciprocal of resistivity) was calculated. XRD is a non-destructive technique primarily used to essentially distinguish the structure of the phases of a crystalline material and can provide further information on crystal structure. The XRD analysis was carried out with a Shimadzu 6000 equipment using a diffractometer with $\mathrm{Cu} K_{\alpha}$ radiation $(\lambda=0.15406 \mathrm{~nm})$. The detector scans along a circle around the sample, in order to collect all the diffracted X-ray beams. For the measurement of temperature dependences of the AC and DC conductivity cell was designed and fabricated. For temperature measurement the highly shielded home-made furnace controlled via type $\mathrm{K}$ thermocouple directly connected to a temperature controller was used. Generally, the temperature control is within $0.01 \mathrm{~K}$ for temperatures less than $30 \mathrm{~K}$ and within $0-05 \mathrm{~K}$ for temperatures less than $100 \mathrm{~K}$ and within $0.2 \mathrm{~K}$ up to $300 \mathrm{~K}$. The change in the resistance with temperature was noted.

\section{Results and discussion}

\subsection{Powder X-ray diffraction analysis}

Figure 2 presents the analysis of the result of XRD pattern of MR. XRD pattern reveals the polycrystalline nature of $\mathrm{MR}$. XRD patterns in the range of $5^{\circ}$ to $90^{\circ}$ $2 \theta$ were carried out at room temperature using $\mathrm{Cu} K_{\alpha}$

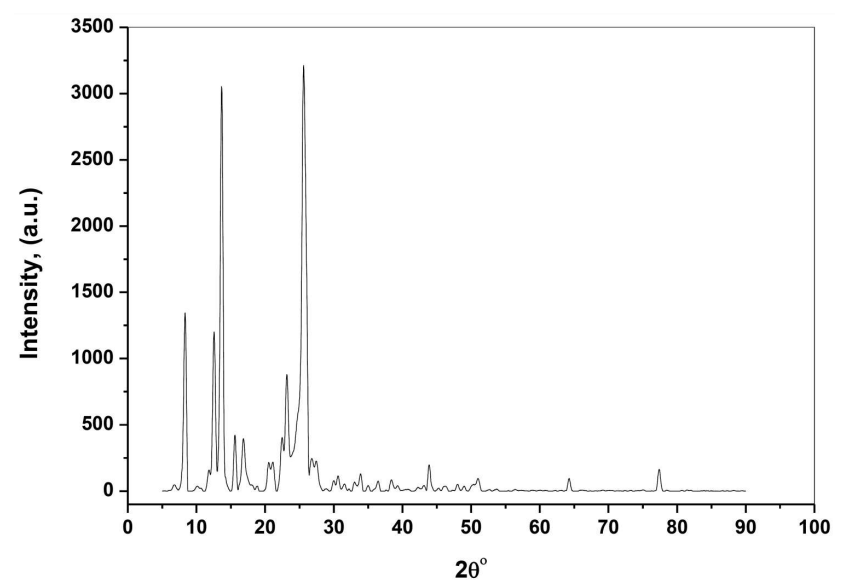

Fig. 2. XRD pattern of MR organic compound.

radiation $(\lambda=0.15418 \mathrm{~nm})$. The following estimation conditions were led: tube voltage of $50 \mathrm{kV}$, tube current of $30 \mathrm{~mA}$, step scan mode with a step size $0.02^{\circ} 2 \theta$ and counting time of $1 \mathrm{~s}$ per step for preliminary examination and $12 \mathrm{~s}$ per step for unit cell parameter refinement. Using crsyfire and checkcell software [13], we found the cell parameters values of MR sample which were indexed and refined.

\subsection{Temperature dependence DC conductivity and Arrhenius plot of the $M R$}

The DC conductivity and resistance are related according to

$$
\sigma=\frac{l}{R A}
$$

where $\sigma$ is conductivity in $\mathrm{S} \mathrm{cm}^{-1}$ or $\Omega^{-1} \mathrm{~cm}^{-1}, l$ is thickness in $\mathrm{cm}, R$ is resistance in $\Omega$ and $A$ is area in $\mathrm{cm}^{2}$.

Figure 3 shows the variation for $\sigma_{\mathrm{DC}}$ of $\mathrm{MR}$ at different temperatures. In the present work, an increase in the DC conductivity with an increase in temperature was observed and would be attributed to high mobility of free charges (i.e., polarons and free ions) at higher temperature, and is fitted to Arrhenius exponential law equation [14], as depicted in Fig. 3:

$$
\sigma=\sigma_{01} \mathrm{e}^{-\Delta E_{1} / k \mathrm{~B} T}+\sigma_{02} \mathrm{e}^{-\Delta E_{2} / k_{\mathrm{B}} T},
$$

where $T$ is the absolute temperature; $\sigma_{01}$ and $\sigma_{02}$ are the high temperature limits of conductivity, for region I and II, respectively, $-\Delta E_{1}$ and $-\Delta E_{2}$ are the DC conduction activation energy (in $\mathrm{J}$ ) of the first and second regions, respectively. $k_{\mathrm{B}} T$ is associated with temperature variation in the measurement where $k_{\mathrm{B}}$ is the Boltzmann constant. The linear regression of the Arrhenius plot $\log \left(\sigma_{D C}\right)$ versus $1000 / T$ gives the slope of $-\Delta E_{1}$ and $-\Delta E_{2}$ values, indicating that there are two conduction mechanisms in the examined range of temperature. The conduction activation energy of the conductivity affected by temperature is the minimum energy required to overcome potential barrier. For both the samples it was seen that there are two straight lines with 


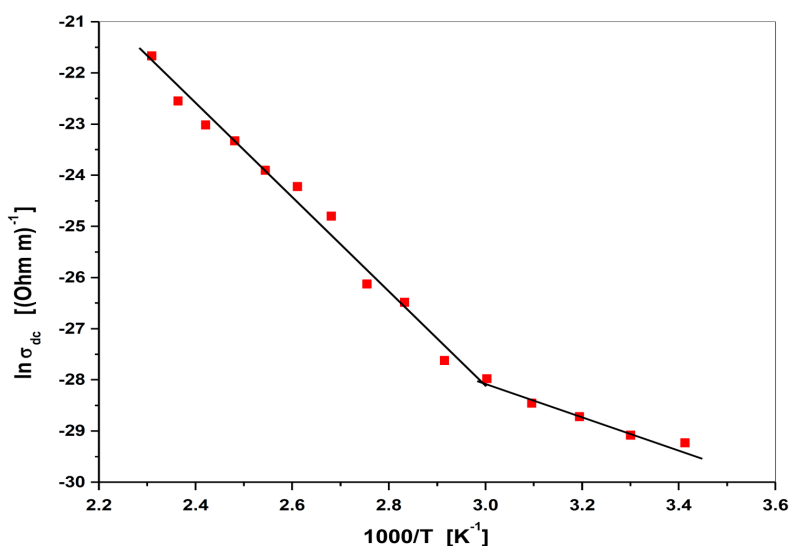

Fig. 3. A semilogarithmic plot of the temperature dependence of the DC electrical conductivity of MR organic compound. The solid lines represent the best linear fits to the data.

differing slopes. This implies that there are two regions of differing activation energies, one for higher temperature area and the other for lower temperature district. The results demonstrated show that there are two types of conduction mechanisms. The activation energies and pre-exponential factors were determined using the leastsquares fit of the experimental data to a straight line via origin software.

The values $\Delta E_{1}$ and $\sigma_{01}$ for region (I) were found to be $0.321 \mathrm{eV}$, and $7.7 \times 10^{-8}(\Omega \mathrm{m})^{-1}$, respectively, and the values $\Delta E_{2}$ and $\sigma_{02}$ for region (II) were determined to be $0.458 \mathrm{eV}$, and $3.11 \times 10^{-5}(\Omega \mathrm{m})^{-1}$, respectively. The calculated band gap values are larger than that of the optical activation energy gap as in intrinsic semiconductors. Temperature-dependence measurements of electrical conductivity reveal that the electrical conduction mechanism is thermally activated process and DC conductivity increases exponentially as temperatures increase over the temperature range studied [15]. The electrical conductivity of all investigated samples was increased with the increase of temperature indicating that the electrical conduction mechanism similar to that of semiconductors.

\subsection{Temperature dependence of $A C$ electrical conductivity of $M R$}

The measured conductance, $G(\omega)$ from $150 \mathrm{~Hz}$ up to $1 \mathrm{MHz}$ was used to calculate $\mathrm{AC}$ conductivity, $\sigma_{\mathrm{AC}}(\omega)$, as shown in Fig. 3, using the following expression [16]:

$\sigma_{\text {Totall AC }}(\omega)=\sigma_{\mathrm{AC}}(\omega)+\sigma_{\mathrm{DC}}(\omega \rightarrow 0)$,

where $\omega$ is the temporal frequency.

The temperature-dependent value of the first part is related to the dielectric relaxation of bound charge carriers (dipoles) as determined from orientational polarization. This accounts for the free charges resident in the bulk and is often taken to be independent of frequency [16]. At higher temperatures, compositions with high conductivity, show the Arrhenius behavior and the

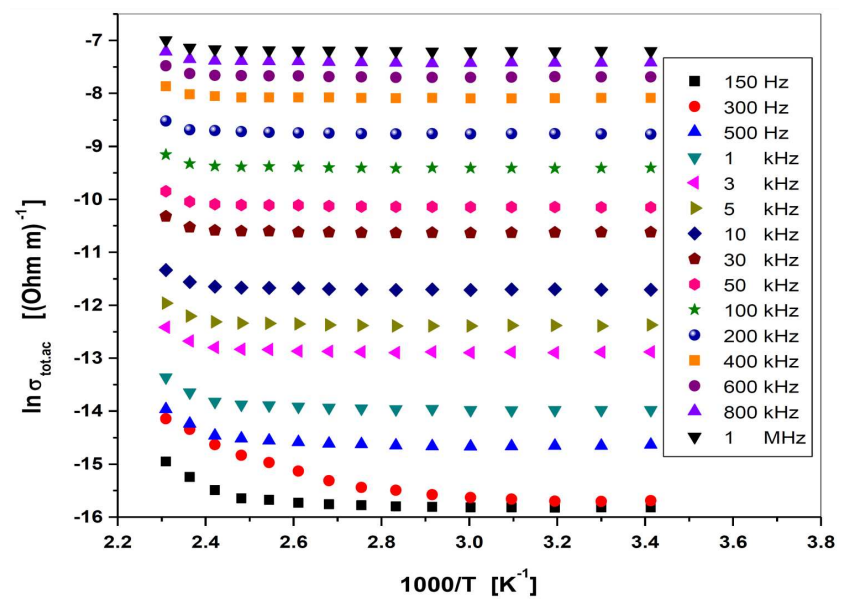

Fig. 4. Temperature dependence of the calculated total electrical conductivities $\sigma_{\text {Totall AC }}(\omega)$ plotted in Arrhenius coordinates at different frequencies for MR organic compound.

variation with frequency is small. At low temperatures, $\mathrm{AC}$ conductivity indicates just weak temperature dependence and somewhat higher. It is clear from Fig. 4 that $\ln \sigma_{\mathrm{AC}}(\omega)$ increases nonlinearly with the temperature especially at low frequency.

By using the values of $\ln \sigma_{\mathrm{AC}}(\omega)$ at different temperatures, the value of activation energy can be calculated. If $\ln \sigma_{\mathrm{AC}}(\omega)$ depends linearly on the temperature with single activation energy, the mechanism is due to the hopping between shallow states close to the mobility edges, where the density of states is taken to be constant but the obtained dependence is not linear. A possible explanation of the behavior is that the density of states is rising continuously toward the band edges instead of being sharp at a specific energy [16]. The same behaviour for frequency and temperature dependence was obtained for perovskite $(\mathrm{Pb}, \mathrm{Ca}) \mathrm{TiO}_{3}$ ceramic in the investigated range [17]. Expression for the AC conductivity has been suggested by Elliott and Rockstad [18, 19], where the AC component of the frequency-dependent conductivity is described as the sum of two main types of conduction mechanisms

$$
\sigma_{\mathrm{AC}}(\omega)=\sigma_{f}+\sigma_{s},
$$

where $\sigma_{f}$ represents relatively weak temperature dependence mechanism, which has been interpreted as being due to bipolaron hopping between $\mathrm{D}^{+}$and $\mathrm{D}^{-}$centers (localized states lie at or around the Fermi level (EF)). $\sigma_{s}$ represents the stronger temperature-dependent mechanism component of AC conductivity, which has been interpreted as being due to excitation to the localized states at the band edges, where the conduction takes place by quantum mechanical tunneling and is obtained by subtraction $\sigma_{f}$ from $\sigma_{\mathrm{AC}}(\omega)$. This behavior of AC conductivity is in agreement with the results obtained before for other amorphous materials. The $\sigma_{\mathrm{AC}}(\omega)$ in $\mathrm{MR}$ organic compound falls below $\sigma_{\mathrm{DC}}$ at higher 


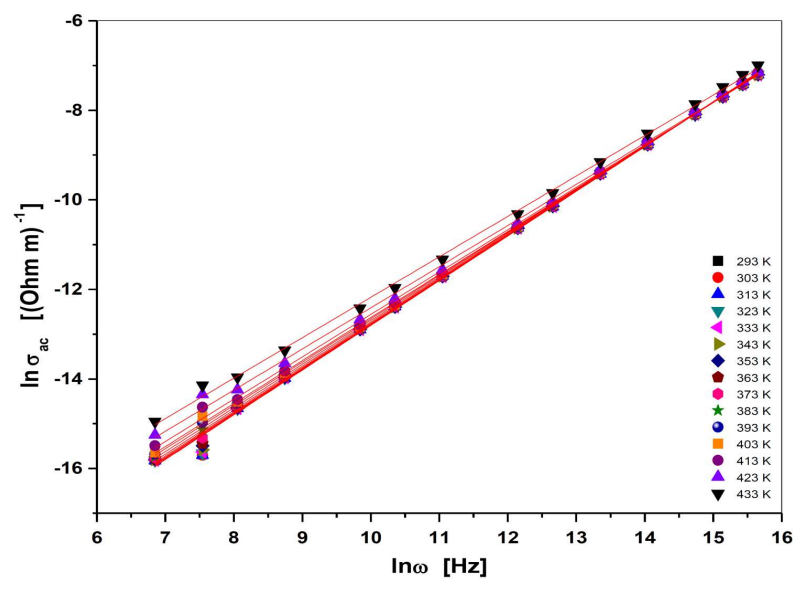

Fig. 5. Frequency dependence of $\sigma_{\mathrm{AC}}(\omega)$ for the MR at different temperatures.

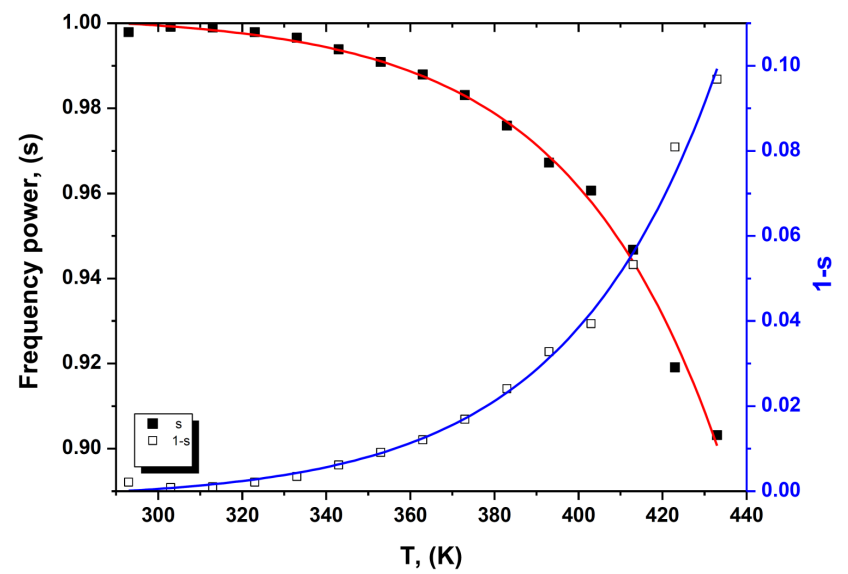

Fig. 6. Temperature dependence of the frequency exponent $s$.

temperatures, indicating that $\sigma_{\mathrm{AC}}$ due to single polarons is smaller than $\sigma_{\mathrm{DC}}$. A single polaron hopping has been evaluated at higher temperatures mechanism. At higher temperatures, donor states $\left(\mathrm{D}^{0}\right)$ states are produced by thermal excitation of $\mathrm{D}^{+}$and $\mathrm{D}^{-}$states and single polaron hopping (i.e., one electron hopping between $\mathrm{D}^{0}$ and $\mathrm{D}^{+}$and a hole between $\mathrm{D}^{0}$ and $\mathrm{D}^{+}$) becomes a dominant process [20].

The dependence of the AC conductivity $\sigma_{\mathrm{AC}}(\omega)$ on the temperature suggests that the dissipation process is thermally activated. The value of the activation energy $\Delta E(\omega)$ is calculated at different frequencies from the slope of lines in Fig. 4 using the Arrhenius law [21-23]:

$$
\sigma_{\mathrm{AC}}(\omega)=\sigma_{0} \exp \left(-\Delta E(\omega) / k_{\mathrm{B}} T\right),
$$

where $\sigma_{0}$ is constant and $\Delta E(\omega)$ is the activation energy for AC electrical conduction mechanism, $k_{\mathrm{B}}$ is the Boltzmann constant, and $T$ is the absolute temperature. The activation energy has been calculated to be $0.23 \mathrm{eV}$.
Figure 5 shows the frequency dependence of AC conductivity $\sigma_{\mathrm{AC}}(\omega)$ for the $\mathrm{MR}$ at the temperature range of 285-435 K. A feature common to all types of amorphous semiconductors is that $\mathrm{AC}$ electrical conductivity $\sigma_{\mathrm{AC}}(\omega)$ increases with increasing frequency according to Eq. (5). The AC conductivity of the sample is expressed with the help of the following equation [24]:

$$
\sigma_{\mathrm{AC}}(\omega)=\sigma_{\text {tot }}(\omega)-\sigma_{\mathrm{DC}}=A(\omega)^{s},
$$

where $\omega$ is the angular frequency and is given by $\omega=2 \pi v, \sigma_{\text {tot }}(\omega)$ is the measured total electrical conductivity, $\sigma_{\mathrm{DC}}$ is the DC electrical conductivity, $s$ is the frequency exponential constant $(s<1)$, and $A$ gives the dependence on the temperature.

The quantity of the frequency exponent $s$ is plotted versus the temperature, as shown in Fig. 6 for the MR. It is clear from this figure that $s$ decreases with increasing temperature, with a constant near-unity at room temperature, and falls within the classically accepted range $0.6<s<1$, which suggests that the frequency dependence of $\sigma_{\mathrm{AC}}(\omega)$ for present material can also be explained by the correlated barrier hopping $(\mathrm{CBH})$ mechanism [25].

\section{Conclusion}

AC and DC electrical conductivity of the organic compound MR dye are intensively investigated. Our samples have been characterized by X-ray diffraction analysis. The DC electrical conductivity of MR exhibited symmetrical behavior to that of the general properties of organic semiconductors, and the mechanism of the AC conductivity of MR is controlled by the correlated barrier hopping model, as confirmed by an observed reduction in activation energy.

\section{References}

[1] J. Lee, S.S. Kim, K. Kim, J.H. Kim, S. Im, Appl. Phys. Lett. 84, 1701 (2004).

[2] R.K. Gupta, R.A. Singh, J. Polym. Res. 11, 269 (2005).

[3] S.R. Forrest, Nature 428, 911 (2004).

[4] J.-L. Brédas, J.E. Norton, J. Cornil, V. Coropceanu, Acc. Chem. Res. 42, 1691 (2009).

[5] J. Kirkpatrick, V. Marcon, J. Nelson, K. Kremer, D. Andrienko, Phys. Rev. Lett. 98, 227402 (2007).

[6] V. Coropceanu, J. Cornil, D.A. da Silva Filho, Y. Olivier, R. Silbey, J.-L. Brédas, Chem. Rev. 107, 926 (2007).

[7] A. Troisi, D.L. Cheung, D. Andrienko, Phys. Rev. Lett. 102, 116602 (2009).

[8] G. Muthuraman, T.T. Teng, Progr. Nat. Sci. 19, 1215 (2009).

[9] T. Kilicoglu, M.E. Aydin, Y.S. Ocak, Physica B 388, 244 (2007).

[10] C. Sahoo, A.K. Gupta, P. Anjali, Desalination 181, 91 (2005). 
[11] H. Lachheb, E. Puzenat, A. Houas, M. Ksibi, E. Elaloui, C. Guillard, J.M. Herrmann, Catal. B Environ. 39, 75 (2002).

[12] S. Salman Ashraf, A. Mahammad, A. Rauf Seham, Dyes Pigments 69, 74 (2006).

[13] A.A.M. Farag, I.S. Yahia, Synth. Met. 161, 32 (2011).

[14] E. Schmidbauer, P. Schmid-Beurmann, J. Solid State Chem. 177, 207 (2004).

[15] Sh.A. Mansour, I.S. Yahia, F. Yakuphanoglu, Dyes Pigments 87, 144 (2010); Sh.A. Mansour, I.S. Yahia, G.B. Sakr, Solid State Commun. 150, 1386 (2010).

[16] S.R. Elliott, Adv. Phys. 36, 135 (1987); N.F. Mott, E.A. Davis, Electronic Processes in Non-Crystalline Materials, Clarendon Press, Oxford 1971.
[17] H.M. El-Mallah, Acta Phys. Pol. A 122, 174 (2012).

[18] S.R. Elliott, Philos. Mag. B 37, 553 (1978).

[19] H.K. Rockstad, Solid State Commun. 1507, 2233 (1971); J. Non-Cryst. Solids 8-10, 621 (1972).

[20] K. Shimakawa, Philos. Mag. B 46, 123 (1982).

[21] S.A. Reda, Dyes Pigments 75, 526 (2007).

[22] Y. Fahrettin, E. Ertan, O. Mustafa, Physica B 382 , 285 (2006).

[23] S.R. Lukic, S.J. Skuban, F. Skuban, D.M. Petrovic, A.S. Tveryanovich, Physica B 403, 2578 (2008).

[24] A.K. Jonscher, Nature 267, 673 (1977).

[25] S.R. Elliott, Adv. Phys. 36, 135 (1987). 